\title{
Sensing Users Selection with Overhead Reduction for Cognitive Wireless Ad-Hoc Networks.
}

\author{
Ferran Adelantado and Angel A. Juan \\ Universitat Oberta de Catalunya (UOC) \\ Barcelona, Spain \\ \{ferranadelantado, ajuanp\}@uoc.edu
}

\author{
Harry Skianis \\ University of the Aegean \\ Samos, Greece \\ cskianis@ aegean.gr
}

\author{
Christos Verikoukis \\ CTTC \\ Castelldefels, Spain \\ cveri@cttc.es
}

\begin{abstract}
Cognitive Wireless Ad-Hoc Networks use cooperation in order to sense the spectrum accurately. However, the space diversity provided by the cooperative sensing is only efficient when the sensing users are selected appropriately. This paper proposes a distributed probability-based mechanism that allows the Secondary Users to decide what channels should be sensed by each user in order to reduce the sensing overhead.
\end{abstract}

\section{INTRODUCTION}

With the spread and deployment of a huge variety of wireless networks during the last decade and the consequent growth of the wireless broadband demand, the efficient use of the scarce radio resources has attracted the interest of the scientific community. In this context, the Federal Communications Commission (FCC) reported in 2002 that the spectrum was underutilized [1], and this underutilization was owing to variations of the spectrum allocation in terms of time, frequency, and geographical location. The possibility of exploiting the underused resources has encouraged the development of networks with cognitive capacity, the socalled Cognitive Wireless Networks (CWN), which are able to discover and reuse the available transmission opportunities.

Cognitive wireless ad-hoc networks are made up of a set of secondary users (SU) that sense the spectrum to detect unused frequency bands/channels. The spectrum sensed by the SUs is licensed to a set of primary users (PU), which have constraintless access. When an unused band is found, SUs coordinate themselves to establish a communication on that channel. SUs are allowed to use the frequency band as long as they do not incur interference on the PUs. When PUs transmissions are resumed, SUs must vacate the licensed band immediately. As the nature of the wireless medium entails undesired phenomena such as the hidden terminal or the exposed node problems, sensing becomes a critical aspect. Space diversity is an effective strategy to combat the unreliability of the sensed information, but it implies the cooperation of the SUs [2]. In cognitive wireless ad-hoc networks, where the lack of infrastructure and the scarcity of radio resources makes more essential to increase the efficiency of the sensing process, there exists a trade-off between the accuracy of the spectrum sensing information and the signaling overhead.

Although cooperative sensing challenges have been addressed extensively with different strategies [3], to the best of our knowledge, the proper selection of the sensing users received little attention. In particular, in the literature works have focused on the selection and/or weighting of the received sensing information when all the SUs sense the same set of channels [4] [5]. The main contribution of this paper is the design of a dynamic mechanism that allows each SU sensing only a subset of the channels. The smart channel selection and the cooperation among SUs guarantee the sensing of all the channels and the adaptability to environment variations, while reduce the amount of information exchange.

The paper is organized as follows: In Section II the motivation and the basis of the problem are stated. In Section III the proposed mechanism is explained. Finally, in Section V the results are discussed. Conclusions are presented in Section VI.

\section{PROBLEM FORMULATION}

Cognitive wireless ad-hoc networks are usually organized in clusters to cooperate [6]. In each cluster, there is usually one of the SUs that undertakes the role of cluster head and coordinates the exchange of sensing information. This cluster head collects and combines the sensing observations from the rest of the SUs in the cluster and makes the decision on the availability of the channels. Then, the cluster head informs the rest of SUs about the decision. As cognitive wireless ad-hoc networks are dynamic in nature, the degree of cooperation among SUs determines the completeness of the available information. Nonetheless, the sensing information load grows as the number of SUs and sensed channels rises. Therefore, the proposed mechanism is intended to reduce the transmission of inaccurate or excessively redundant information.

Assume a cognitive wireless ad-hoc network comprised of a set of secondary users, $U=\left\{u_{k}: 1 \leq k \leq K\right\}$. The spectrum is also divided into a set of channels. Let us define $F=\left\{f_{m}: 1 \leq m \leq M\right\}$ as the set of channels to be sensed. The channels $f_{m} \in F$ are licensed to PUs, and they are sensed periodically by $u_{k} \in U$. The set of channels to be sensed, $F$, is selected by the cluster head and distributed to all the SUs in the cluster through a Common Control Channel, not licensed to PUs and where all SUs content to gain transmission opportunities. The Common Control Channel is denoted by $f_{0}$ -i.e. $f_{0} \notin F$ - and it is not interfered by PUs transmissions. In order to reduce the sensing overhead, each SU selects and senses only $N$ out of the $M$ channels, with $N<M$. In other words, each SU only senses a subset of the channels 
selected by the cluster head. By doing so, all SUs experience a reduction of the time and energy consumption. The objective will be achieved if, notwithstanding the reduction of the cooperation degree, the accuracy of the cluster head decisions is maintained.

Regarding the algorithm used by the cluster head to select the set of channels for sensing, $F$, the reader is referred to [7] and [8] for some examples.

Hereafter we propose a mechanism for each SU to perform the selection of the $N$ channels.

\section{MECHANISM DESCRIPTION}

\section{A. Estimation of the sensing accuracy}

First, we define $\theta_{k, m}$ as the accuracy of the information obtained by $u_{k}$ from channel $f_{m}$. Specifically, $\theta_{k, m}=1$ if $u_{k}$ is able to detect the presence of PUs transmitting on $f_{m}$ and $\theta_{k, m}=0$ if it is not due to distance, fading, etc. As $\theta_{k, m}$ is unknown for $u_{k}$, each SU must estimate it. The estimation is denoted by $\theta_{k, m}^{*}$.

We focus now on a channel $f_{m}$. Assume a sensing process is triggered at time $t_{j}$. If a user $u_{k}$ senses $f_{m}$ during the sensing process triggered at time $t_{j}$, it generates the observation $s_{k, m}\left(t_{j}\right)$. This observation, $s_{k, m}\left(t_{j}\right)$, is the result of sensing $f_{m}$ and may be 0 if no PU was detected and 1 otherwise. All the SUs that sense $f_{m}$ during the sensing process generate an observation and deliver it to the cluster head through the Common Control Channel $f_{0}$. The protocol used to transmit $s_{k, m}\left(t_{j}\right)$ from $u_{k}$ to the cluster head is out of the scope of this work, but an example may be found in [9]. Upon the reception of all the observations, the cluster head combines/fuses them all. There are several fusion rules that may be used to combine the observations, but the most common rules are the OR and the AND operations [10]. The result of fusing all the collected observations of $f_{m}$ is denoted by $g_{m}\left(t_{j}\right)$, and it is also distributed to all the users in $U$ through the Common Control Channel. Accordingly, by definition, $g_{m}\left(t_{j}\right)$ may be 0 or 1 .

Notice that, if $\theta_{k, m}=1$, there will be rare situations where $s_{k, m}\left(t_{j}\right)$ and $g_{m}\left(t_{j}\right)$ differ. On the contrary, there will be big differences between $s_{k, m}\left(t_{j}\right)$ and $g_{m}\left(t_{j}\right)$ very often if $\theta_{k, m}=0$. We define $\varphi_{k, m}\left(t_{j}\right)$ as the sum of the differences occurred between the observations of $u_{k}$ and the decisions of the cluster head during the last $W$ sensing processes in which $u_{k}$ sensed $f_{m}$,

$$
\varphi_{k, m}\left(t_{j}\right)=\sum_{n=0}^{W-1}\left|s_{k, m}\left(t_{j}-\Delta t_{k, m, n}\right)-g_{m}\left(t_{j}-\Delta t_{k, m, n}\right)\right|
$$

where $\Delta t_{k, m, n}$ is the time elapsed between $t_{j}$ and the $n$th previous sensing process in which $f_{m}$ was sensed by $u_{k}$. For instance, assume that $u_{k}$ senses a channel $f_{m}$ at time $t_{j}, t_{j}-\tau_{1}$ and $t_{j}-\tau_{2}$ (with $\tau_{1}<\tau_{2}$ ). Then, when $\varphi_{k, m}\left(t_{j}\right)$ is calculated, $\Delta t_{k, m, 0}=0, \Delta t_{k, m, 1}=\tau_{1}$ and $\Delta t_{k, m, 2}=\tau_{2}$. According to the definition in (1), we may estimate $\theta_{k, m}^{*}=1$ for large $\varphi_{k, m}\left(t_{j}\right)$ values and $\theta_{k, m}^{*}=0$ otherwise. Consequently, if a threshold $\varphi_{t h}$ is set, $\theta_{k, m}^{*}=1$ when $\varphi_{k, m}\left(t_{j}\right)<\varphi_{t h}$, and $\theta_{k, m}^{*}=0$ otherwise.

\section{B. Considerations about the estimation of the accuracy}

The estimation of the accuracy, $\theta_{k, m}^{*}$, is subject to the false alarm probability, $P_{f a}$, and the misdetection probability, $P_{m d}$, as well as to the activity of the PUs. With regard to false alarm and misdetection, the proposed mechanism mitigates their effect on $\theta_{k, m}^{*}$ by using $\varphi_{k, m}\left(t_{j}\right)$ and $\varphi_{t h}$. Thus, the probability of erroneous estimation decreases as $W$ and $\varphi_{t h}$ grow. However, it is also true that the delay between a change in $\theta_{k, m}$ and the corresponding detection in $\theta_{k, m}^{*}$ rises with $W$ and $\varphi_{t h}$. As for the activity of the PUs, notice that it is impossible to detect whether $\theta_{k, m}$ is 1 or 0 if the PU transmitting on $f_{m}$ is silent, since $g_{m}\left(t_{j}\right)=s_{k, m}\left(t_{j}\right)=0$ even if $\theta_{k, m}=0$. Accordingly, if this situation lasts longer than $\left(W-\varphi_{t h}\right)$ sensing processes in which $u_{k}$ senses $f_{m}, \theta_{k, m}^{*}$ may be erroneously set to 1 . Yet, this is not a serious problem, since such an erroneous accuracy estimation does not entail interference on PUs transmissions.

\section{Channels selection}

When a sensing process is triggered, each user $u_{k}$ selects $N$ out of the $M$ channels contained in $F$. In order to minimize the amount of inaccurate sensing information, the $N$ channels selected by $u_{k}$ should have $\theta_{k, m}=1$. However, as $\theta_{k, m}$ is unknown, the estimation $\theta_{k, m}^{*}$ is used instead of $\theta_{k, m}$ and the $N$ selected channels should accomplish $\theta_{k, m}^{*}=1$. Although the selection mechanism should guarantee that the selected channels have $\theta_{k, m}^{*}=1$, the mechanism should also be able to adapt to the propagation variations and detect when an inaccurate channel turns into accurate. Therefore, the channels with $\theta_{k, m}^{*}=0$ should also be sensed to check whether, for a user $u_{k}$, they are still inaccurate or not.

The mechanism proposed in this study is a probability-based method. Let us define $\lambda_{k, m}$ as a weighting factor for a channel $f_{m}$. The probability that a user $u_{k}$ selects a channel $f_{m}$ will be proportional to $\lambda_{k, m}$. As the mechanism is intended to reduce the amount of inaccurate information, the probability that a user $u_{k}$ selects a channel $f_{m}$ with $\theta_{k, m}^{*}=1$ should be higher than the probability that $u_{k}$ selects a channel $f_{m^{\prime}}$ with $\theta_{k, m^{\prime}}^{*}=0$. Accordingly, $\lambda_{k, m}>\lambda_{k, m^{\prime}}$.

We define $\lambda_{k, m}=\theta_{k, m}^{*}(\gamma-1)+1$, where $\gamma$ is a positive real number such that $\gamma>1$. Hence, $\lambda_{k, m}=1$ when $\theta_{k, m}^{*}=0$ inaccurate observations- and $\lambda_{k, m}=\gamma$ when $\theta_{k, m}^{*}=1$-accurate observations-. Now, we denote the set of channels sensed by $u_{k}$ during the sensing process triggered at time $t_{j}$ by $F_{k}\left(t_{j}\right)$. As $u_{k}$ selects the channels from $F$, then $F_{k}\left(t_{j}\right) \subseteq F$. By definition, the complement of set $F_{k}\left(t_{j}\right)$ relative to set $F$, denoted by $F_{k}^{C}\left(t_{j}\right)$, contains the channels not selected yet by $u_{k}$. Thus, the probability that a user $u_{k}$ senses a channel $f_{m} \in F_{k}^{C}\left(t_{j}\right)$, is denoted by $\pi_{k, m}\left(t_{j}\right)$ and defined as

$$
\pi_{k, m}\left(t_{j}\right)=\frac{\lambda_{k, m}}{\sum_{\substack{m^{\prime} \\ f_{m^{\prime}} \in F_{k}^{C}\left(t_{j}\right)}} \lambda_{k, m^{\prime}}}
$$

In practice, $u_{k}$ selects the $N$ channels as follows: 


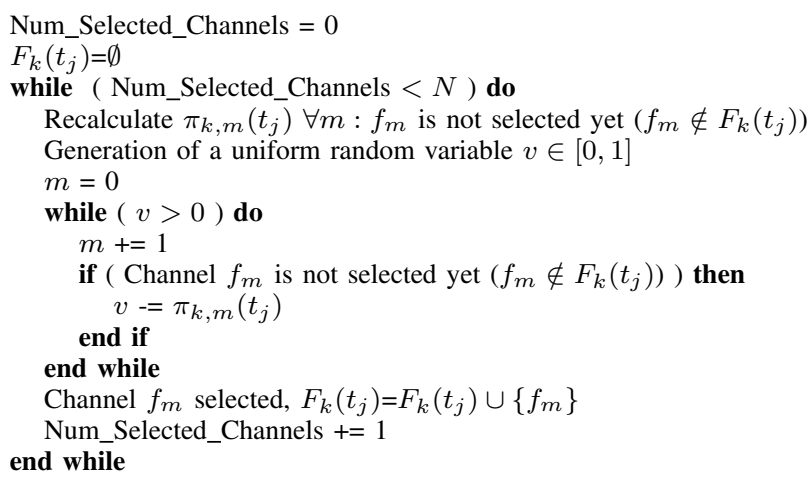

\section{Mechanism scalability}

The number of packets devoted to share the sensing information grows with the number of SUs, namely $K$. Consequently, it might be surmised that the redundancy of the information transmitted from the cluster members to the cluster head also rises.

The process described hereafter is aimed at limiting the number of SUs that transmit the sensing observations to the cluster head. By doing this, it is assured that the sensing overhead remains below a threshold. In particular, the cluster head defines a minimum number of SUs, $K_{\min }$, that should share the sensing observations with the cluster head. The rest of the users, even though all of them sense the spectrum, do not transmit their observations to the cluster head. The rationale behind this strategy is twofold: on the one hand the overhead caused by the transmission of the SUs sensing information is reduced; on the other hand the SUs that do not transmit their sensing information can, however, update the accuracy estimations as described in Section III-A. Each SU transmits the sensing observations with a probability $P_{t x}$, where $P_{t x}$ may be expressed as

$$
P_{t x}= \begin{cases}0 & \text { if } K \leq K_{\min } \\ \frac{K_{\min }}{K} & \text { otherwise }\end{cases}
$$

The method used to decide whether a SU shares the information or not is equivalent to the method explained in Section III-C. In this case, each SUs generates a random variable uniformly distributed between 0 and 1 , e.g. $v$, and transmits if $v \leq P_{t x}$. Both parameters, $K_{\min }$ and $P_{t x}$, are decided and distributed during the cluster setup phase.

Up to now, the Common Control Channel, i.e. $f_{0}$, has been assumed error-free and collision-free. Now, we denote by $P_{\text {loss }}$ the probability that the transmission of an observation from a cluster member to the cluster head is erroneous or collides. According to the description, for a given number of non cluster head SUs, $(K-1)$, the number of users that finally transmit the observations, denoted by $\widehat{K}$, follows a binomial distribution $\widehat{K} \sim B\left(K-1, P_{t x}\right)$. Likewise, the number of observations received correctly by the cluster head, denoted by $\widetilde{K}$, also follows a binomial distribution $\widetilde{K} \sim B\left(\widehat{K}, 1-P_{\text {loss }}\right)$ [11]. Taking this into account, the probability mass function $P(\widetilde{K}=$ $\widetilde{k}$ ) may be derived with some algebra and is given by

$$
\begin{aligned}
P(\widetilde{K}=\widetilde{k})= & \sum_{\substack{i=\widetilde{k}\\
}}^{K-1}\left[C_{\widetilde{k}}^{i} C_{i}^{K-1} \cdot\left(1-P_{\text {loss }}\right)^{\widetilde{k}} \cdot P_{\text {loss }}^{i-\widetilde{k}} .\right. \\
& \left.P_{t x}^{i} \cdot\left(1-P_{t x}\right)^{K-1-i}\right]
\end{aligned}
$$

where $C_{\widetilde{k}}^{i}$ and $C_{i}^{K-1}$ are binomial coefficients [11]. Equation (4) shows that the number of SUs that share their observations without collision or error, $\widetilde{K}$, and whose observations are combined by the cluster head, may be adjusted by varying $K_{\min }$

\section{Analysis of the Mechanism}

\section{A. Sensing overhead}

In this Section, an analysis of the sensing overhead is stated. The total Common Control Channel capacity is given by $R_{t}$ bps. We denote the time between two consecutive sensing processes by $T_{s}$. Accordingly, the sensing overhead may be expressed as $R_{s}=\frac{L_{s}}{T_{s}}$ bps, where $L_{s}$ stands for the sensing information (expressed in bits) exchanged by the cluster members within $T_{s}$. In this Section $P_{t x}=1$ is assumed. With this assumption the expressions obtained hereafter consider the worst case, i.e. when the mechanism causes the maximum sensing overhead (all the cluster members transmit their observations).

First, the cluster head broadcasts a packet that contains PHY and MAC header bits, $L_{h 1}$, and the identifiers of the channels in $F$. If $L_{c}$ bits are required to identify a single channel, the packet length is $L_{h 1}+M L_{c}$. Secondly, each user of the cluster senses $N$ channels. If the time required to sense a single channel is $t_{c}$, the time devoted to sense the $N$ channels is equivalent to the time required to transmit a packet with a length $N t_{c} R_{t}$ bits. Then, all the users except the cluster head transmit the information coordinately. As mentioned in Section III-A, the protocol used to coordinate the SUs transmissions is out of the scope of this study, but the reader is referred to [9] for examples. In case no collisions occur, the number of bits transmitted to the cluster head is $(K-1)\left(L_{h 2}+N L_{d}\right)$, where $L_{h 2}$ is the length of the PHY and MAC layer header and $L_{d}$ is the number of bits required to map a single channel identifier and the associated sensing observation.

Accordingly, the sensing overhead when the mechanism is applied, $R_{s}^{N}$, is given by

$$
R_{s}^{N}=\frac{1}{T_{s}}\left(L_{h 1}+M L_{c}+N t_{c} R_{t}+(K-1)\left(L_{h 2}+N L_{d}\right)\right)
$$

When the mechanism is not applied, all the users in the cluster except the cluster head sense the $M$ channels. The sensing overhead in this case is $R_{s}^{M}$

$$
R_{s}^{M}=\frac{1}{T_{s}}\left(L_{h 1}+M L_{c}+M t_{c} R_{t}+(K-1)\left(L_{h 2}+M L_{d}\right)\right)
$$

The reduction of the sensing overhead when the mechanism is applied is calculated as $\Delta R_{s}=\left(R_{s}^{M}-R_{s}^{N}\right) / R_{s}^{M}$.

According to (5) and (6), the obtained overhead reduction is dependent on $K, M$ and $N$. Then, if a minimum overhead 
reduction is intended, $\Delta R_{s m i n}$, the following condition should be accomplished

$$
\Delta R_{\sin } \leq \Delta R_{s}
$$

Deriving (7), the minimum $N$ required to achieve a minimum sensing overhead reduction $\Delta R_{s m i n}$ is given by

$N \leq M\left(1-\Delta R_{s m i n}\right)-\Delta R_{s m i n} \frac{L_{h 1}+M L_{c}+(K-1) L_{h 2}}{t_{c} R_{t}+(K-1) L_{d}}$

\section{B. Metrics to assess the mechanism}

As exposed in Section III-C, each SU senses $N$ channels within a sensing period. The selection of the channels is carried out in a probability-based manner, and therefore there exists a probability that the selection is not accurate. For instance, if channel $f_{m}$ is selected, the probability that $\theta_{k, m}=0$ is not null. In order to asses the mechanism operation, we denote by $N_{1}$ the number of channels selected by a user $u_{k}$ with $\theta_{k, m}=1$. Analogously, $N_{0}$ refers to the selected channels with $\theta_{k, m}=0$, and by definition $N=N_{0}+N_{1}$. Consequently $P\left(N_{1}=0\right)$ is the probability that none of the $N$ observations carried out by a SU is actually accurate.

The observations of the SUs are affected by the false alarm and misdetection probabilities, denoted by $P_{f a}$ and $P_{m d}$ respectively. As a consequence of the combination process, the information distributed by the cluster head presents cluster false alarm and misdetection probabilities, different from $P_{f a}$ and $P_{m d}$, denoted by $Q_{f a}$ and $Q_{m d}$. Whereas $Q_{f a}$ may diminish the capacity of the cognitive wireless ad-hoc network, $Q_{m d}$ may cause interference on the PUs. The two probabilities should be reduced, but particularly $Q_{m d}$.

Finally, each SU updates the accuracy estimations according to the described mechanism. Let us define $\Theta$ as the number of channels with a correct estimation $\left(\theta_{k, m}=\theta_{k, m}^{*}\right)$. The expected value of $\Theta$, namely $E[\Theta]$, shows the quality of the estimations and the capacity of monitoring several channels. If $E[\Theta]$ is large, the mechanism is able to maintain good estimations for several channels thereby reducing the cluster misdetection probability.

\section{RESUlts AND DisCUSSION}

In the simulated scenario 5 licensed channels are considered, $F\left\{f_{m}: m=1, \cdots, 5\right\}$. The activities of the PUs on the channels are modeled as i.i.d. ON-OFF random processes. The probability that a channel which is in the $\mathrm{ON}(\mathrm{OFF})$ state remains in the same state is denoted by $P_{O n O n}\left(P_{O f f O f f}\right)$. With regard to the accuracy of the observations of a channel $f_{m}$ sensed by a user $u_{k}$, the variable $\theta_{k, m}$ is also modeled as an ON-OFF process with a probability $P_{00}$ (or $P_{11}$ ) of remaining in the same state when $\theta_{k, m}=0$ (or $\theta_{k, m}=1$ ). In the simulations, $P_{O n O n}=P_{O f f O f f}=0.8$ and $P_{11}=P_{00}=0.95$. It is worth noting that the probability that the channel is in the ON or OFF state is 0.5. However, once the channel is in a state, it is more likely to remain in the same state rather than change to the other. The same occurs with the accuracy. The accuracy is assumed to change more slowly than the PUs activity. The considered fusion rule is the OR operation. Each simulation consists in analyzing 500 consecutive sensing processes. The results are obtained by averaging 1000 simulations.

Fig. 1 plots $P\left(N_{1}=0\right)$, the probability that none of the channels selected by $u_{k}$ has $\theta_{k, m}=1$. The number of channels sensed by each user is $N=2$, and $W=3$. There are $K=20$ SUs in the cluster, $P_{t x}=1$ and $P_{\text {loss }}=0$. It is assumed that all the SUs can communicate each other and that the cluster head only collects and distributes the sensing information, as well as makes the decision, but does not sense the spectrum. The link between all the SUs, $f_{0}$, is considered to be error-free.

Fig. 1 shows the impact of $\gamma$ on $P\left(N_{1}=0\right)$. Notice that when $\gamma \rightarrow 1$, all the channels are sensed by a user $u_{k}$ with the same probability regardless of $\theta_{k, m}^{*}$, since $\lambda_{k, m}=1$. As $\gamma$ grows, $P\left(N_{1}=0\right)$ decreases and tends to a stable value. This is due to the fact that, when $\gamma \gg 1, \pi_{k, m}\left(t_{j}\right) \gg \pi_{k, m^{\prime}}\left(t_{j}\right)$ with $\theta_{k, m}^{*}=1$ and $\theta_{k, m^{\prime}}^{*}=0$. In this situation, according to the results plotted in Fig. 1, $\gamma$ has little impact on $P\left(N_{1}=0\right)$ for large $\gamma$.

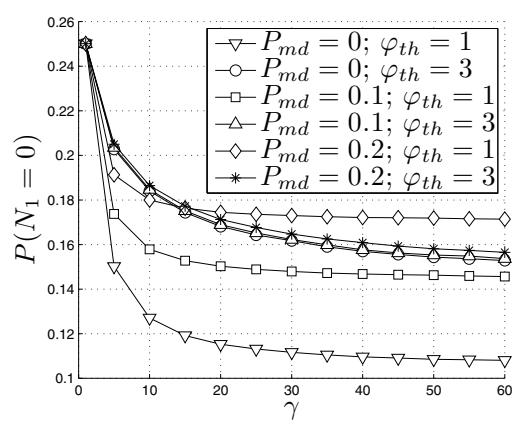

Fig. 1. Probability that none of the channels selected by a user has $\theta_{k, m}=1$ $(K=20, N=2, W=3$ )

In Fig. 2 the cluster misdetection probability , $Q_{m d}$, is shown. As expected, $Q_{m d}$ and $P\left(N_{1}=0\right)$ are tightly coupled. Hence, the lower $P\left(N_{1}=0\right)$ is, the lower $Q_{m d}$ results. It is also worth noting that the impact of $\varphi_{t h}$ on $Q_{m d}$ depends on $P_{m d}$. When $P_{m d}$ is low, large $\varphi_{t h}$ values cause higher $Q_{m d}$ levels than when $\varphi_{t h}$ values are small. On the contrary, when $P_{m d}$ is high, $Q_{m d}$ presents a minimum for medium $\varphi_{t h}$ values. The reason for such a behavior lies in the delay and reliability of the estimation $\theta_{k, m}^{*}$. If the sensing of a channel $f_{m}$ is reliable $\left(P_{m d} \rightarrow 0\right)$, a large $\varphi_{t h}$ increases the time elapsed between a change in $\theta_{k, m}$ and the corresponding $\theta_{k, m}^{*}$ adaptation, thereby worsening $P\left(N_{1}=0\right)$ and $Q_{m d}$. When $P_{m d}$ grows, $\varphi_{t h}$ prevents the user from wrong estimations due to unreliable sensing, and therefore it counteracts the negative effect of the delay increase. However, when $\varphi_{t h} \rightarrow W$, the excessive increase of the delay in the detection does affect negatively the cluster misdetection probability.

The number of SUs within the cluster is determinant in the operation of the cognitive network. In Fig.'s 3, 4 and 5 the evolution of $P\left(N_{1}=0\right), Q_{m d}$ and $E[\Theta]$ as a function of the 


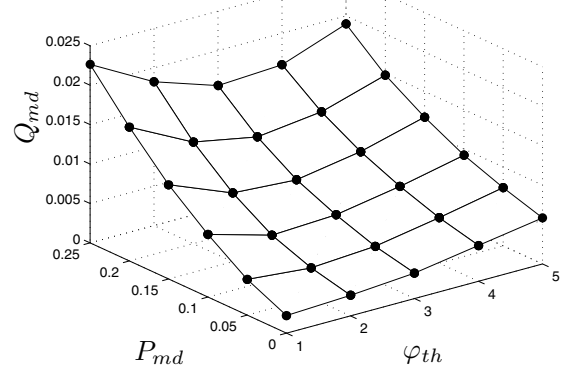

Fig. 2. Cluster misdetection probability with $K=20, \gamma=30$ and $W=5$.

number of SUs is plotted. For all the figures $M=5, N=2, \gamma=30$, $W=3, \varphi_{t h}=2$ and $P_{l o s s}=0.2$. Three different scenarios are exposed: when $P_{t x}=1$ (i.e. $K_{\min }$ larger than any $K$, labeled as $K_{\min }=\mathrm{N} / \mathrm{A}$ ), when $K_{\min }=5$ and when $K_{\min }=10$.

As it is shown in Fig. 3, when the cluster is composed of few SUs, the knowledge of the environment is limited and the reliability of the accuracy estimation is low. As $K$ grows, the knowledge of the spectrum is improved and $P\left(N_{1}=0\right)$ tends to a stable value. When the number of SUs that transmit their sensing information is limited, it also has, as expected, an impact on $P\left(N_{1}=0\right)$. Thus, such a reduction in the number of transmitting SUs, $\widehat{K}$, is translated into a stagnation or a tiny increase of $P\left(N_{1}=0\right)$.

The same effect may be observed in Fig. 4 and Fig. 5, where $Q_{m d}$ and $E[\Theta]$ are plotted. By setting $K_{m i n}$, it is possible to reduce the sensing overhead, but at the same time the minimum $Q_{m d}$ and the maximum $E[\Theta]$ are also impacted. The appropriate $K_{\min }$ should be then chosen according to such a trade-off.

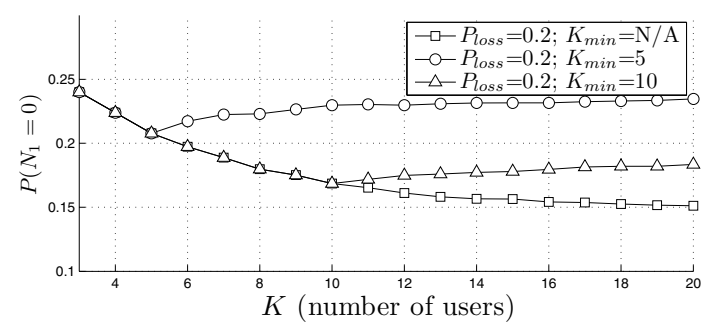

Fig. 3. Probability that none of the channels selected by a user has $\theta_{k, m}=1$ $\left(\gamma=30, P_{m d}=0, N=2, W=3\right.$ and $\left.\varphi_{t h}=2\right)$.

\section{CONCLUSIONS}

The proposed mechanism is aimed at reducing the sensing process overhead by properly selecting the sensing users. The mechanism has shown that an appropriate sensing user selection may be carried out by distributing the decision and using local and global information. As a consequence users are able to classify the spectrum channels according to the accuracy of the measurement and diminish the amount of shared information. The results have shown that the sensing

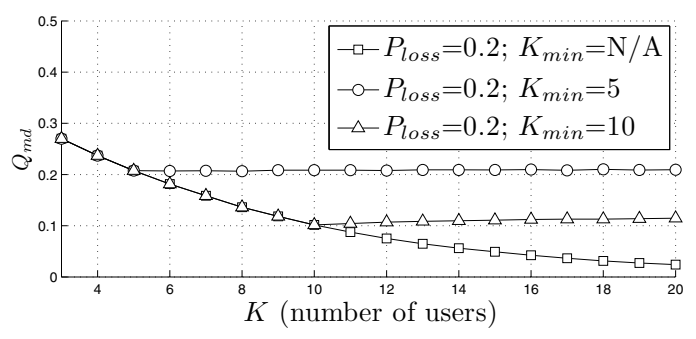

Fig. 4. Cluster misdetection probability $\left(\gamma=30, P_{m d}=0, N=2, W=3\right.$ and $\left.\varphi_{t h}=2\right)$.

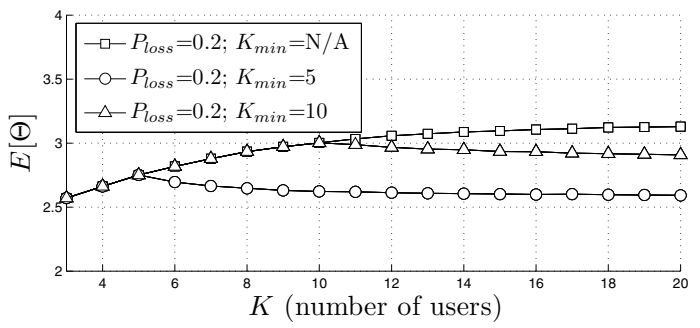

Fig. 5. Average number of channels with correct accuracy estimation $(\gamma=30$, $P_{m d}=0, N=2, W=3$ and $\left.\varphi_{t h}=2\right)$.

overhead may be reduced while maintaining good misdetection probability. Additionally, the scalability of the mechanism has also been assured by limiting the number of SUs that transmit sensing information on the Common Control Channel.

\section{REFERENCES}

[1] "Spectrum policy task force report," Federal Communications Commission (FCC), FCC 02-155 86-194, Nov. 2002.

[2] T. Yucek and H. Arslan, "A survey of spectrum sensing algorithms for cognitive radio applications," vol. 11, no. 1, pp. 116-130, 2009.

[3] D. Niyato and E. Hossain, "Cognitive radio for next-generation wireless networks: An approach to opportunistic channel selection in ieee 802.11based wireless mesh," IEEE Wireless Communications, vol. 16, no. 1 , pp. 46-54, 2009.

[4] M. Matsui, H. Shiba, K. Akabane, and K. Uehara, "A cooperative sensing technique with weighting based on distance between radio stations," in Proc. APCC '08, Tokyo, Japan, Oct. 14-16, 2008, pp. 1-4.

[5] Y. Sun, H. Hu, F. Liu, H. Yi, and X. Wang, "Selection of sensing nodes in cognitive radio system based on correlation of sensing information," in Proc. WiCOM '08, Dalian, China, Oct. 12-14, 2008, pp. 1-6.

[6] T. Chen, H. Zhang, G. M. Maggio, and I. Chlamtac, "Cogmesh: A cluster-based cognitive radio network," in Proc. IEEE DySPAN '07, Dublin, Ireland, Apr. 17-20, 2007, pp. 168-178.

[7] H. Kim and K. G. Shin, "Fast discovery of spectrum opportunities in cognitive radio networks," in Proc. IEEE DySPAN '08, Chicago, USA, Oct. 14-17, 2008, pp. 1-12.

[8] B. Hamdaoui, "Adaptive spectrum assessment for opportunistic access in cognitive radio networks," vol. 8, no. 2, pp. 922-930, 2009.

[9] H. Su and X. Zhang, "Cognitive radio based multi-channel mac protocols for wireless ad hoc networks," in Proc. IEEE Globecom '07, Washington, USA, Apr. 17-20, 2007, pp. 4857-4861.

[10] K. B. Letaief and W. Zhang, "Cooperative communications for cognitive radio networks," in Proc. of the IEEE, Washington, USA, Apr. 17-20, 2007, pp. 4857-4861.

[11] W. D. Wallis, A. P. Street, and J. S. Wallis, Combinatorics, ser. Lecture Notes in Mathematics. Springer, 1972, no. 292. 